\title{
EFFECT OF EPITAXIAL LAYER THICKNESS ON BUILT-IN ELECTRIC FIELD IN REGION OF AlGaAs/SI-GaAs INTERFACE: A PHOTOREFLECTANCE STUDY
}

\author{
T.J. OCHALSKI, J. ŻUK \\ Institute of Physics, M. Curie-Skłodowska University \\ Pl. M. Curie-Skłodowskiej 1, 20-031 Lublin, Poland \\ AND L.A. Vlasukova \\ Institute of Applied Physics, State University, Minsk, Belarus
}

\begin{abstract}
We present a study of detailed line shapes of photoreflectance spectra for $\mathrm{Al}_{0.3} \mathrm{Ga}_{0.7} \mathrm{As} / \mathrm{SI}-\mathrm{GaAs}$ epitaxial layers grown by MBE. All measurements were performed at $80 \mathrm{~K}$ under UHV conditions with a special care for the samples surface quality. A set of the photoreflectance spectra was collected for photon energies close to the $\mathrm{GaAs}$ and $\mathrm{Al}_{0.3} \mathrm{Ga}_{0.7} \mathrm{As}$ band gaps $\left(E_{0}\right)$. The photoreflectance spectra originated in the vicinity of the $\mathrm{Al}_{0.3} \mathrm{Ga}_{0.7} \mathrm{As} / \mathrm{SI}-\mathrm{GaAs}$ interface were analyzed using the complex Airy function model of Franz-Keldysh oscillations. To examine the effect of the epitaxial layer thickness on parameters characterizing the interface, a step-by-step chemical etching was applied for stripping the top layers. The built-in electric field intensity, field inhomogeneity and phenomenological broadening parameter for interface regions were determined as a function of the epilayer thickness.
\end{abstract}

PACS numbers: 78.66.Fd, 78.20.--e

\section{Introduction}

Photoreflectance (PR) spectroscopy is an important contactless modulation technique, widely used for investigating semiconducting materials and epitaxial multilayers [1-9]. PR method can be used for evaluation of a number of important band structure parameters, alloy compositions and doping concentrations. PR data display spectral features near critical point energies, which in the intermediate-field limit [1] may be explained by a perturbation in the dielectric function of a solid due to the presence of electric field (Franz-Keldysh effect). It has been shown $[2,3]$ that in a typical PR experiment, i.e., in the case of low amplitude ac modulation, the observed Franz-Keldysh oscillations (FKOs) originate from built-in surface 
or interface static electric fields. Relatively little attention has been paid to the investigation of these fields by means of the PR technique in a combination with the step-etching method [4]. The main objective in this work is to evaluate the electric field parameters for the $\mathrm{Al}_{0.3} \mathrm{Ga}_{0.7} \mathrm{As} / \mathrm{SI}-\mathrm{GaAs}$ interface by a careful analysis of the PR spectra.

\section{Experimental}

$\mathrm{Al}_{0.3} \mathrm{Ga}_{0.7} \mathrm{As}$ epilayers of a $0.6 \mu \mathrm{m}$ thickness were grown by MBE on SI [100] $\mathrm{GaAs}$ substrates. The nominal $\mathrm{Al}$ composition agreed within the experimental uncertainty with the one evaluated from the energy $E_{0}$ of the direct $\mathrm{Al}_{0.3} \mathrm{Ga}_{0.7} \mathrm{As}$ gap, as measured by PR. The PR apparatus consisted of a SPM-2 grating monochromator with a $200 \mathrm{~W}$ halogen lamp providing the probe beam and an $\mathrm{Ar}^{+}$laser $(\lambda=488.0 \mathrm{~nm})$ used as the pump light source of a $5 \mathrm{~mW}$ power. The pump and probe beams, both of about $2 \mathrm{~mm}$ diameters, overlap on the sample surfaca. The samples were kept in a UHV chamber $\left(p \approx 10^{-9} \mathrm{mbar}\right)$ of a liquid $\mathrm{N}_{2}$ optical cryostat.

A step-by-step chemical etching was carried out at RT in a solution consisting of $1\left(\mathrm{H}_{2} \mathrm{SO}_{4}\right): 2\left(\mathrm{H}_{2} \mathrm{O}_{2}\right): 200\left(\mathrm{H}_{2} \mathrm{O}\right)$ by volume. This etchant provided a mirror-like quality of the surface. The etch rate was $c a .1 \mathrm{~nm} / \mathrm{s}$, as determined by an interferometric microscope from the step's height measurement between the etched and protected parts of the sample. Immediately after the etching, the sample was put into the UHV chamber and the PR measurements were performed.

Displayed in Fig. 1 there are the PR spectra at $80 \mathrm{~K}$ of the $\mathrm{Al}_{0.3} \mathrm{Ga}_{0.7} \mathrm{As} /$ SI-GaAs sample: before the etching (the upper one) and after some subsequent

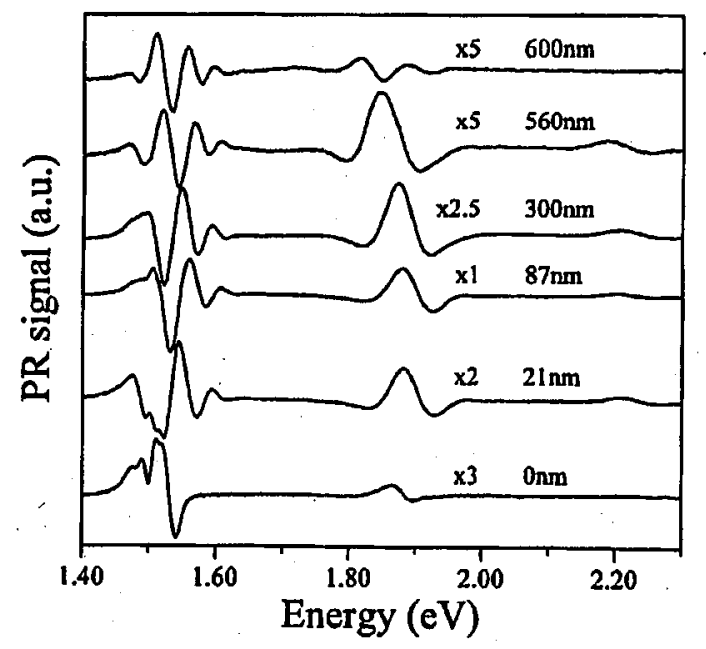

Fig. 1. Experimental PR spectra of $\mathrm{Al}_{0.3} \mathrm{Ga}_{0.7} \mathrm{As} / \mathrm{SI}-\mathrm{GaAs}$ sample at $80 \mathrm{~K}$, collected at different etching steps. The numbers indicate the thickness of the remaining epitaxial layer after each etching. 
stages of the chemical treatment. Franz-Keldysh oscillations are clearly visible in the spectra in the vicinity of the GaAs $E_{0}$ gap $(1.51 \mathrm{eV})$, with an exception of the last (bottom) spectra, collected for the completely uncovered SI-GaAs substrate. The shape of the spectrum changes there and has a typical low-field third derivative functional form (TDFF), proposed by Aspnes [1]. In addition, a feature at energy slightly below the band gap is observed for some spectra in Fig. 1. The extrema of the FKO shift up in energy as the etching is continued, what can be interpreted in terms of optical interference phenomena [5]. At photon energies near the $\mathrm{Al}_{0.3} \mathrm{Ga}_{0.7} \mathrm{As}$ band gap $E_{0}(\approx 1.84 \mathrm{eV})$ no FKOs are observed. The shape of this part of the spectrum can be described by the TDFF. Its origin is probably two-fold and the (weak) contribution from the spin-orbit split valence band of GaAs is not excluded. This is supported by the presence of the PR signal from a bare GaAs substrate (the bottom spectrum in Fig. 1). At about $2.2 \mathrm{eV}$ a very weak PR feature can be noticed, which mostly probably corresponds to the $E_{0}+\Delta_{0}$ critical point of $\mathrm{Al}_{0.3} \mathrm{Ga}_{0.7} \mathrm{As}$ layer. Both PR signatures of the epitaxial layer completely disappear for the uncovered GaAs substrate.

\section{Discussion and conclusion}

We decided to perform a complete line shape analysis in the intermediate-field limit $[1,6,7]$, using the Airy functions to fit the PR spectra in the vicinity of the band gap energy $E_{0}$. The usual method of the FKO extrema plots provides a direct measure of the built-in electric field [3], but some serious drawbacks accompanied [4], can lead to the erroneous estimations of the electric field. Our program computes complex Airy functions using a table of the Bessel functions and performs curve fittings with a help of a modified Levenberg-Marquardt procedure. The model takes into account contributions to the spectra both from the light and heavy hole valence bands. The effect of lifetime broadening is included via the phenomenological parameter $\Gamma$. Batchelor et al. [7] suggested an energy-dependent form $\Gamma=\Gamma_{0} \exp \left[\left(\hbar \omega-E_{0}\right) \delta\right]$, where $\Gamma_{0}$ is the nominal broadening at the band gap energy and $\delta$ is the electric field inhomogeneity factor. The parameter $\delta$ has been shown to include the effects of a field gradient and modulation between two finite fields [8].

An example of PR spectra with FKO features in the GaAs $\left(E_{0}\right)$ energy region is shown in Fig. 2, together with the best fit to the experimental data. This spectrum corresponds to a $125 \mathrm{~nm}$ thick $\mathrm{Al}_{0.3} \mathrm{Ga}_{0.7} \mathrm{As}$ layer. The fit is very satisfactory throughout the analyzed energy range. For some spectra, however, fits are worse due to the presence of additional weak features below $E_{0}$ (Fig. 1), resembling the PR lines attributed to excitonic transitions in GaAs. According to Estrera et al. [8] they originate from the portions of the space-charge region, where the electric fields are low enough not to quench the exciton [3]. Figure 3 shows the $\mathrm{Al}_{0.3} \mathrm{Ga}_{0.7} \mathrm{As}$ layer thickness dependences of electric field intensity, the broadening parameter $\Gamma$, and the electric field inhomogeneity factor $\delta$. All three parameters have been obtained from the fits to sixteen PR spectra, collected after consecutive etchings. The electric field intensity increases up to the value of $48 \mathrm{kV} / \mathrm{cm}$ with the decrease in the AlGaAs layer thickness. The maximum field intensity is achieved for the substrate covered with a $45 \mathrm{~nm}$ thick epilayer. Subsequently, a rapid decrease 


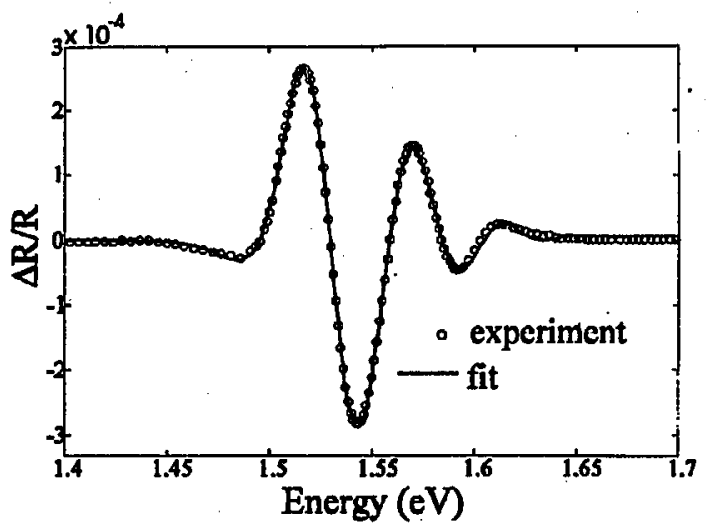

Fig. 2. The PR spectrum of $\mathrm{Al}_{0.3} \mathrm{Ga}_{0.7} \mathrm{As} / \mathrm{SI}-\mathrm{GaAs}$ at $80 \mathrm{~K}$, with strong Franz-Keldysh oscillations in the vicinity of the GaAs band gap $E_{0}$. The fit includes two Airy profiles corresponding to light and heavy holes.

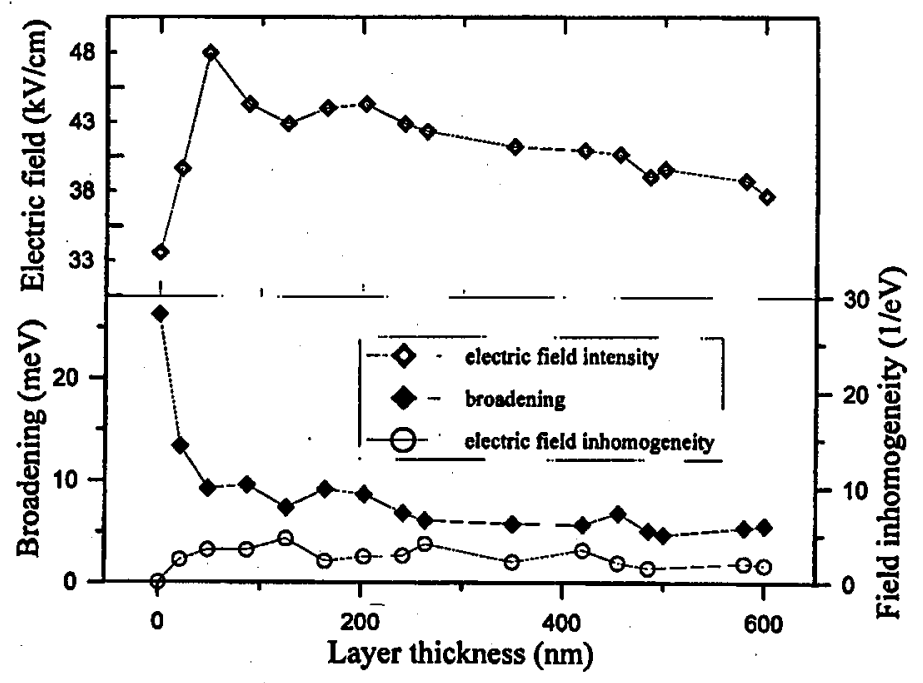

Fig. 3. Built-in electric field intensity $\varepsilon$, broadening parameter $\Gamma$, and electric field inhomogeneity factor $\delta$ as a function of the epitaxial $\mathrm{Al}_{0.3} \mathrm{Ga}_{0.7}$ As layer thickness.

in the field is observed, approaching the $\mathrm{Al}_{0.3} \mathrm{Ga}_{0.7} \mathrm{As} / \mathrm{SI}-\mathrm{GaAs}$ interface and a weaker electric field corresponding to the SI-GaAs surface is detected, that is connected with a disappearance of the FKO. This is in agreement with the work of Sydor et al. [9], where the FKO signals were generated in the PR after a MBE growth of a $180 \mathrm{~nm}$ thick layer of the same composition on SI-GaAs substrate.

In conclusion, we have shown that the intermediate-field Franz-Keldysh oscillations in the PR spectrum of the $\mathrm{Al}_{0.3} \mathrm{Ga}_{0.7} \mathrm{As} / \mathrm{SI}-\mathrm{GaAs}$ interface are related 
to a superposition of the surface and the interface electric fields. The influence of the surface potential is important even for relatively thick AlGaAs epilayers.

\section{References}

[1] D.E. Aspnes, Surf. Sci. 37, 418 (1973).

[2] R.N. Bhattacharya, H. Shen, P. Parayanthal, F.H. Pollak., T. Coutts, H. Aharoni, Phys. Rev. B 37, 4044 (1988).

[3] H. Shen, F.H. Pollak, J.M. Woodall, J. Vac. Sci. Technol. B 8, 413 (1990).

[4] P.J. Hughes, B.L. Weiss, T.J.C. Hosea, J. Appl. Phys. 77, 6472 (1995).

[5] T.J.C. Hosea, P.J. Hughes, B.L. Weiss, J. Appl. Phys. 77, 2672 (1995).

[6] B.O. Seraphin, N. Bottka, Phys. Rev. 145, 628 (1966).

[7] R.A. Batchelor, A.C. Brown, A. Hamnett, Phys. Rev. B 41, 1401 (1990).

[8] J.P. Estrera, W.M. Duncan, R. Glosser, Phys. Rev. B 49, 7281 (1994).

[9] M. Sydor, J. Angelo, J.J. Wilson, W.C. Mitchel, M.-Y. Yen, Phys. Rev. B 40, 8473 (1989). 